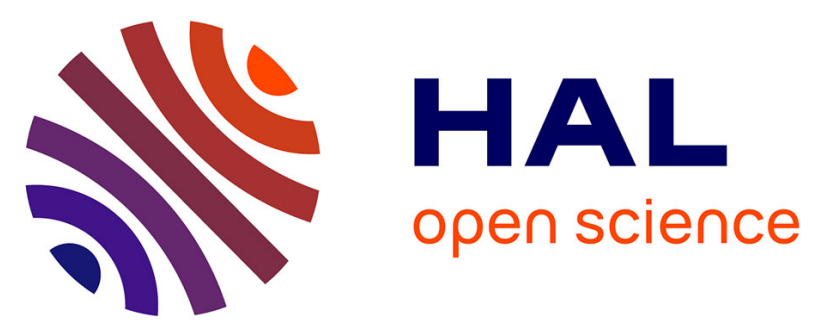

\title{
Comparative proteomic analysis of human mesenchymal and embryonic stem cells: towards the definition of a mesenchymal stem cell proteomic signature
}

Stéphane Roche, B. Delorme, R. Oostendorp, R. Barbet, C. Caton, Danièle Noël, K. Boumediene, H. Papadaki, B. Cousin, C. Crozet, et al.

\section{To cite this version:}

Stéphane Roche, B. Delorme, R. Oostendorp, R. Barbet, C. Caton, et al.. Comparative proteomic analysis of human mesenchymal and embryonic stem cells: towards the definition of a mesenchymal stem cell proteomic signature. Proteomics, 2009, 9 (2), pp.223-232. 10.1002/pmic.200800035 . hal00357170

\author{
HAL Id: hal-00357170 \\ https://hal.science/hal-00357170
}

Submitted on 18 Dec 2017

HAL is a multi-disciplinary open access archive for the deposit and dissemination of scientific research documents, whether they are published or not. The documents may come from teaching and research institutions in France or abroad, or from public or private research centers.
L'archive ouverte pluridisciplinaire HAL, est destinée au dépôt et à la diffusion de documents scientifiques de niveau recherche, publiés ou non, émanant des établissements d'enseignement et de recherche français ou étrangers, des laboratoires publics ou privés. 


\title{
Comparative proteomic analysis of human mesenchymal and embryonic stem cells: Towards the definition of a mesenchymal stem cell proteomic signature
}

\author{
Stephane Roche ${ }^{1,2}$, Bruno Delorme ${ }^{3 *}$, Robert A. J. Oostendorp ${ }^{4 *}$, Romain Barbet ${ }^{5^{*}}$, \\ David Caton $^{6 *}$, Daniele Noel ${ }^{7,8}$, Karim Boumediene ${ }^{9}$, Helen A. Papadaki ${ }^{10}$, \\ Beatrice Cousin ${ }^{6}$, Carole Crozet ${ }^{1}$, Ollivier Milhavet ${ }^{1}$, Louis Casteilla $^{6}$, Jacques Hatzfeld ${ }^{5}$, \\ Christian Jorgensen ${ }^{7,8}$, Pierre Charbord ${ }^{3}$ and Sylvain Lehmann ${ }^{1,2,8}$ \\ ${ }^{1}$ CNRS, Institut de Génétique Humaine, UPR1142, Montpellier, France \\ ${ }^{2}$ CHU Montpellier, Laboratoire de Biochimie, Hôpital St. Eloi, Montpellier, France \\ ${ }^{3}$ Laboratoire d'Hématopoïèse, INSERM/ESPRI EA 3855, Faculté de Médecine de Tours, France \\ ${ }^{4} 3^{\text {rd }}$ Department of Internal Medicine, Klinikum rechts der Isar, \\ Technische Universität München, München, Germany \\ ${ }^{5}$ Institut André Lwoff, CNRS, UPR 9045, Villejuif, France \\ ${ }^{6}$ Institut Louis Bugnard, UMR 5241, UPS, CNRS, Toulouse, France \\ ${ }^{7}$ INSERM U844, Montpellier, France \\ ${ }^{8}$ Université Montpellier 1, Montpellier, France \\ ${ }^{9}$ Laboratoire Matrice Extracellulaire et Pathologie, UFR de Médecine, EA3214, \\ Université de Caen Basse-Normandie, Caen, France \\ ${ }^{10}$ Department of Hematology, University of Crete School of Medicine, Heraklion, Crete, Greece
}

\begin{abstract}
Mesenchymal stem cells (MSC) are adult multipotential progenitors which have a high potential in regenerative medicine. They can be isolated from different tissues throughout the body and their homogeneity in terms of phenotype and differentiation capacities is a real concern. To address this issue, we conducted a 2-DE gel analysis of mesenchymal stem cells isolated from bone marrow (BM), adipose tissue, synovial membrane and umbilical vein wall. We confirmed that BM and adipose tissue derived cells were very similar, which argue for their interchangeable use for cell therapy. We also compared human mesenchymal to embryonic stem cells and showed that umbilical vein wall stem cells, a neo-natal cell type, were closer to BM cells than to embryonic stem cells. Based on these proteomic data, we could propose a panel of proteins which were the basis for the definition of a mesenchymal stem cell proteomic signature.
\end{abstract}

Keywords:

Adipose tissue / Bone marrow / Embryonic stem cell / Mesenchymal stem cell / Umbilical vein

Correspondence: Professor Sylvain Lehmann, CNRS, Institut de Génétique Humaine, UPR1142, F-34000 Montpellier, France E-mail: sylvain.lehmann@igh.cnrs.fr

Fax: +33-4-99-61-99-31

Abbreviations: ADSC, adipose derived stem cells; BM, bone marrow; hESC, human embryonic stem cells; MSC, mesenchymal stem cells; PMF, peptide mass fingerprint; SynoSC, synovial membrane-derived stem cells; UVSC, umbilical vein derived somatic cells

\section{Introduction}

Regeneration takes place in the body in different organs following injury or disease. However, adult tissues such as bone, cartilage, tendons, blood vessels and cardiac muscle have a limited capacity for self-repair which leads to nonfunctional scar tissue. In this context, mesenchymal stem

* All these authors contributed equally to the work. 
cells (MSC) which are adult multipotential progenitors of mesoderm cells (osteoblasts, chondrocytes, adipocytes and stroma cells [1]) represent a major hope for tissue-engineered replacement and regenerative medicine. Cells with MSC differentiation abilities can be isolated from bone marrow (BM-MSC), as well, as from a variety of tissues including adipose derived stem cells (ADSC) [2], synovial membrane-derived stem cells (SynoSC) [3, 4], umbilical cord blood [5], cartilage [6] and umbilical vein derived somatic cells (UVSC) [7]. In vitro, culture and differentiation of human MSC before therapeutic use, often require nonphysiological manipulation that might induce some risk for the patients. For example, differentiation of MSC into cardiomyocyte was induced by 5 -azacytidine, an anticancer drug [8]. In the field of regenerative medicine, human embryonic stem cells (hESC) represent also an important hope as they can differentiate into cardiomyocytes, endothelial cells, $\beta$-pancreatic cells and many other tissue types [9]. Both human MSC and hESC share selfrenewal capacity and differentiation into functional tissues. The self-renewal and differentiation potential of human MSC and hESC are however quite different: MSC are multipotent, when hESC are pluripotent. In addition, hESC are isolated from blastocyst and are defined by strict and specific criteria, whereas "MSC like cells" can be isolated from all range of sources and their homogeneity in terms of phenotype and differentiation capacities represent a real issue. To address these differences, the genomic or proteomic approaches could be used to have a general phenotype profile of the cells. The first proteomic analysis of MSC aiming at characterizing subpopulations with various differentiation properties was published in 2001 by Prockop and coworkers [10]. In this study, and in subsequent ones $[11,12]$, mostly focussing on ADSC and BMMSC, the conclusion was that these two cell types were very close together with, however, some differences that were difficult to pinpoint [13-17]. As a matter of fact, from the combined analysis of the published human MSC proteomic studies, it has not been possible to identify a clear proteomic signature specific for MSC [18]. One of the reasons is the different paradigms, protein extraction protocols and analytical approaches used in these studies. Moreover, no study involving cells cultured in different laboratories and multi-site comparison was performed. Therefore, combination of these results to generate a common MSC proteomic signature is a task altered by too many biases.

In the present proteomic work, we compared human MSC isolated from different sources and cultured in strict controlled conditions, with respect to their morphology, expansion and multilineage differentiation capacity. We could define a common proteomic profile that distinguished human MSC from hESC. We also concluded a high proteomic similarity between BM-MSC and ADSC, and we observed that UVSC were closer to the other human MSC than to hESC.

\section{Materials and methods}

\subsection{Cell cultures}

Human BM-MSC, ADSC, UVSC, SynoSC (all from human tissues) and hESC were cultured in optimal conditions to preserve differentiation potentials (Table 1 of Supporting Information). Cells were cultured in different laboratories as follows: P. Charbord and H. A. Papadaki for BM-MSC, L. Casteilla for ADSC, R. Oostendorp for UVSC, C. Jorgensen for SynoSC, J. Hatzfeld for hESC and K. Boumediene for primary human chondrocyte. The presence of the common CD cell markers was confirmed in each laboratory by FACS analysis (Table 1). Each laboratory collected the cell using the same batch of lysing solution and sent the extracts in dry ice for proteomics analysis as described below.

\subsection{Whole cell extract}

To improve the reproducibility of the analysis, protein extraction was performed directly in the culture dishes. Briefly, the cells were washed twice with PBS containing a cocktail of protease inhibitors (P8340, Sigma) then lysed directly in the culture dish with $200 \mu \mathrm{L}$ of lysing buffer $(8 \mathrm{M}$ urea, $2 \mathrm{M}$ thiourea, 4\% CHAPS, $50 \mathrm{mM}$ DTT). The cell lysates were collected and stored at $-80^{\circ} \mathrm{C}$ until use. Protein concentration of the lysates was determined using the PlusOne 2-D Quant Kit (GE Healthcare) following the supplier's recommendations.

\subsection{2-DE}

Proteins were separated on the bases of two dimensions: $\mathrm{pI}$ and masses. For the first dimension, proteins were added to $250 \mu \mathrm{L}$ of rehydration buffer $(9.8 \mathrm{M}$ urea $4 \%$ CHAPS $50 \mathrm{mM}$ DTT and 0.5\% IPG buffer 3-10). IPG strips (13 cm), covering a $\mathrm{pH}$ range of 3-10 were rehydrated with this solution during $9 \mathrm{~h}$ at $20^{\circ} \mathrm{C}$ covered by low viscosity paraffin oil. For focalization, the following voltage/time profile was used: $200 \mathrm{~V}$ for

Table 1. Phenotypes of the stem cells

\begin{tabular}{|c|c|c|c|c|c|}
\hline & BM-MSC & ADSC & SynoSC & UVSC & $\mathrm{hESC}$ \\
\hline CD90 & ++ & ++ & ++ & ++ & ++ \\
\hline CD73 & ++ & ++ & ++ & ++ & - \\
\hline CD105 & ++ & ++ & ++ & ++ & - \\
\hline CD44 & ++ & ++ & ++ & ++ & ++ \\
\hline CD106 & ++ & - & ++ & - & - \\
\hline CD45 & - & - & - & - & - \\
\hline CD34 & - & ++ & - & & - \\
\hline CD56 & - & $+1-$ & - & ++ & - \\
\hline CD31 & - & - & ND & - & - \\
\hline CD166 & ++ & ++ & ++ & ++ & ++ \\
\hline
\end{tabular}

Expression of various markers was tested by flow cytometry $(++$ strongly positive, + positive, - negative, ND not done). 
$1 \mathrm{~h}, 1000 \mathrm{~V}$ for $1 \mathrm{~h}, 3000 \mathrm{~V}$ for $1 \mathrm{~h}$, a gradient between 3000 and $8000 \mathrm{~V}$ during $2 \mathrm{~h}$ and $8000 \mathrm{~V}$ for $5 \mathrm{~h}$. A total of $76000 \mathrm{~V} \cdot \mathrm{h}$ was used. Focused strips were frozen at $-20^{\circ} \mathrm{C}$. For the second dimension, strips were equilibrated for $30 \mathrm{~min}$ in $6 \mathrm{M}$ urea, $30 \%$ glycerol, $2 \% \mathrm{SDS}, 50 \mathrm{mM}$ Tris $\mathrm{pH} 8.8,1 \%$ DTT and then for an additional $30 \mathrm{~min}$ in the same solution except that DTT was replaced by $5 \%$ iodoacetamide. After equilibration, proteins were separated in the second dimension by an SDS-PAGE method using $12 \%$ acrylamide gel with an acrylamide/bisacrylamide ratio of 37.5:1.

To optimize the 2-DE, different quantities of proteins per gel were tested $(10,20$ and $30 \mu \mathrm{g})$. An amount of $20 \mu \mathrm{g}$ was selected since it resulted in the best resolution (total number of spots detected) and the best reproducibility.

\subsection{Silver staining and scan}

Gels were stained with a silver nitrate procedure [19] and scanned at 300 dots per inch using the Labscan 3 software (GE Healthcare) after a procedure of calibration using the kaleidoscope LaserSoft Imaging (Kodak Ref: R020123). Spot detection and quantitation were performed with ImageMaster 2-DE Platinum software (GE Healthcare) and Progenesis SameSpot (Non Linear). The volume of each spot (integrated OD) was calculated as the product of spot area and spot intensity. To take into account experimental variations, 2-D gels were normalized to the volume of all spots detected on the 2-DE gel.

\subsection{Identification of protein expression and statistical test}

The quantification was performed using Progenesis SameSpot by an integration of the grey level of each spot and normalization with the total spot volume. In this method, the volume of each spot is divided by the total volume of all of the spots in the gel.

Three to five replicates with independent samples were performed to ensure reproducibility of the results. For comparison, the BM-MSC gel was used as reference.

Statistical analysis was performed using the Hierarchical Clustering Explorer 3.0 software [20], with an average linkage hierarchical clustering algorithm, using the centred Manhattan Distance coefficient as the similarity metric. Differential function analysis was performed using the Ingenuity Software. A fold increase ratio of 1.7 and a $p$-value of 0.05 calculated by Progenesis SameSpot were used to define the limit of significance.

Data were analysed through the use of Ingenuity Pathways Analysis (Ingenuity ${ }^{\circledR}$ Systems, www.ingenuity.com). The functional analysis identified the biological functions and/or diseases that were most significant to the dataset. Genes from the dataset that met the differential value cut-off of 1.7 and a $p$-value cut-off of 0.05 and were associated with biological functions and/or diseases in the Ingenuity Pathways Knowledge Base were considered for the analysis.

Fischer's exact test was used to calculate a $p$-value determining the probability that each biological function and/or disease assigned to that data set is due to chance alone.

\subsection{Molecular weight and iso-electric point calibration}

The experimental molecular weight and $\mathrm{pHi}$ were obtained using a 2-DE protein standard (BioRad, Richmond, CA, USA): an additional 2-DE gel was performed with a mixture of $10 \mu \mathrm{g}$ of whole MSC extract and $5 \mu \mathrm{L} 2$-DE standard. Additional spots were positioned and annoted with their respective MW and $\mathrm{pI}$. After a step of comparison with this gel, the software calibrates all homologous gels with $\mathrm{pHi}$ and MW.

\subsection{Protein isolation and identification by MS}

Protein spots were excised from coomassie stained 2-DE gels containing $300 \mu \mathrm{g}$ of proteins. Spots were washed in $15 \mu \mathrm{L}$ of $100 \mathrm{mM} \mathrm{NH}_{4} \mathrm{HCO}_{3}$ during $10 \mathrm{~min}$. After addition of $15 \mu \mathrm{L}$ of ACN for $10 \mathrm{~min}$, supernatants were removed and the procedure repeated. After evaporation to dryness in a benchtop SpeedVac, spots were rehydrated in $10 \mu \mathrm{L}$ of trypsin solution $\left(15 \mathrm{ng} / \mu \mathrm{L}\right.$, Promega) and digested overnight at $25^{\circ} \mathrm{C}$ in $10 \mu \mathrm{L}$ of $100 \mathrm{mM} \mathrm{NH}_{4} \mathrm{HCO}_{3} 5 \mathrm{mM} \mathrm{CaCl}_{2}$ buffer. The tryptic peptides were extracted in a two-step procedure: the first step was composed by an addition of $10 \mu \mathrm{L}$ of $100 \mathrm{mM}$ $\mathrm{NH}_{4} \mathrm{HCO}_{3}$ followed by $10 \mathrm{~min}$ of $10 \mu \mathrm{L} \mathrm{ACN}$. This step was repeated twice and supernatants pooled. The second step was a 10 min incubation with $10 \mu \mathrm{L}$ of $5 \%$ formic acid and followed by an addition of $10 \mu \mathrm{L}$ of $\mathrm{ACN}$ for $10 \mathrm{~min}$. This step was repeated twice and the supernatants pooled. Samples were evaporated to dryness and put back in solution in $20 \%$ formic acid and desalted on Millipore ZipTip C18 column.

Peptide masses were determined in the positive-ion reflector mode in an Ultraflex mass spectrometer (Bruker). Peptide mass fingerprints (PMFs) were compared to mammalian databases (Swiss-Prot and Trembl) using MASCOT (http://www.matrixscience.com/cgi/search_form.pl?FORM$\mathrm{VER}=2 \& \mathrm{SEARCH}=\mathrm{PMF}$ ) and Aldente (http://www.expa sy.org/tools/aldente/) algorithms with one missing trypsin cleavage site and a mass deviation smaller than $20 \mathrm{ppm}$. The probabilistic score of MASCOT/Aldente was required for the identification of an unnamed protein.

\section{Results}

\subsection{CD phenotypes of stem cells}

All stem cells were cultured in optimal conditions using adapted media (Table 1 of Supporting Information) to pre- 
serve the multipotency of human MSC and the pluripotency of hESC. The ability to differentiate into adipocyte, osteocyte and chondrocyte lineages was experimentally confirmed before proteomic and phenotypic analysis (data not shown). The classical human MSC phenotype (CD90+ CD73+ $\mathrm{CD} 105+\mathrm{CD} 44+\mathrm{CD} 45-)$ was validated for BM-MSC, ADSC, SynoSC and UVSC (Table 1). As expected, hESC had a distinct phenotypic pattern with the absence of CD73 and CD105 while some markers were cell specific: ADSC expressed CD34 whereas UVSC lacked CD106.

\subsection{Construction of a human MSC 2-DE master gel}

Following the optimization of sample preparation and 2-DE procedures (see Section 2), the primary human BM-MSC were used to generate a human MSC master gel (Fig. 1). Since calculated $\mathrm{pHi}$ and $\mathrm{MW}$ are not reliable parameters following 2-DE, the master gel was calibrated using purified proteins. After silver staining a total of 845 spots was detected (Table 3 of Supporting Information). The pHi distribution of the spots was trimodal, with a maximum around 4.75, 6.25 and 8 while the MW distribution showed that most of the spots were detected between 30 and $40 \mathrm{kDa}$ (Fig. 1 of Supporting Information). Altogether, 231 spots were identified using PMF, representing 164 different proteins, 25\% of the total number of spots and $34 \%$ of the total volume detected (Table 2 of Supporting Information). Cytoplasmic and cell membrane proteins were identified, as well as proteins from mitochondria and nucleus. Post-translationally modified proteins (which were detected as multiple spots) belonged mainly to cytoplasmic and ER compartments, rather than to the mitochondrion and the nucleus. Identified proteins were involved in a large range of functions including: signal transduction, intracellular trafficking, DNA associated proteins, glycolysis, metabolism, catabolism, folding, biosynthesis and splicing (Table 2 of Supporting Information).

\subsection{2-DE of the different stem cells and statistical analysis}

Extracts from the different cell lines were used to carry out 2-DE, as illustrated in Fig. 2. Following a thorough analysis using Progenesis SameSpot (Table 3 of Supporting Information) and the Hierarchical Cluster Explorer software, different stem cell proteomic clusters were detected (Fig. 3). Manhattan distance statistical tests indeed revealed the presence of two main clusters: one including BM-MSC, ADSC, SynoSC and UVSC; the second one being composed

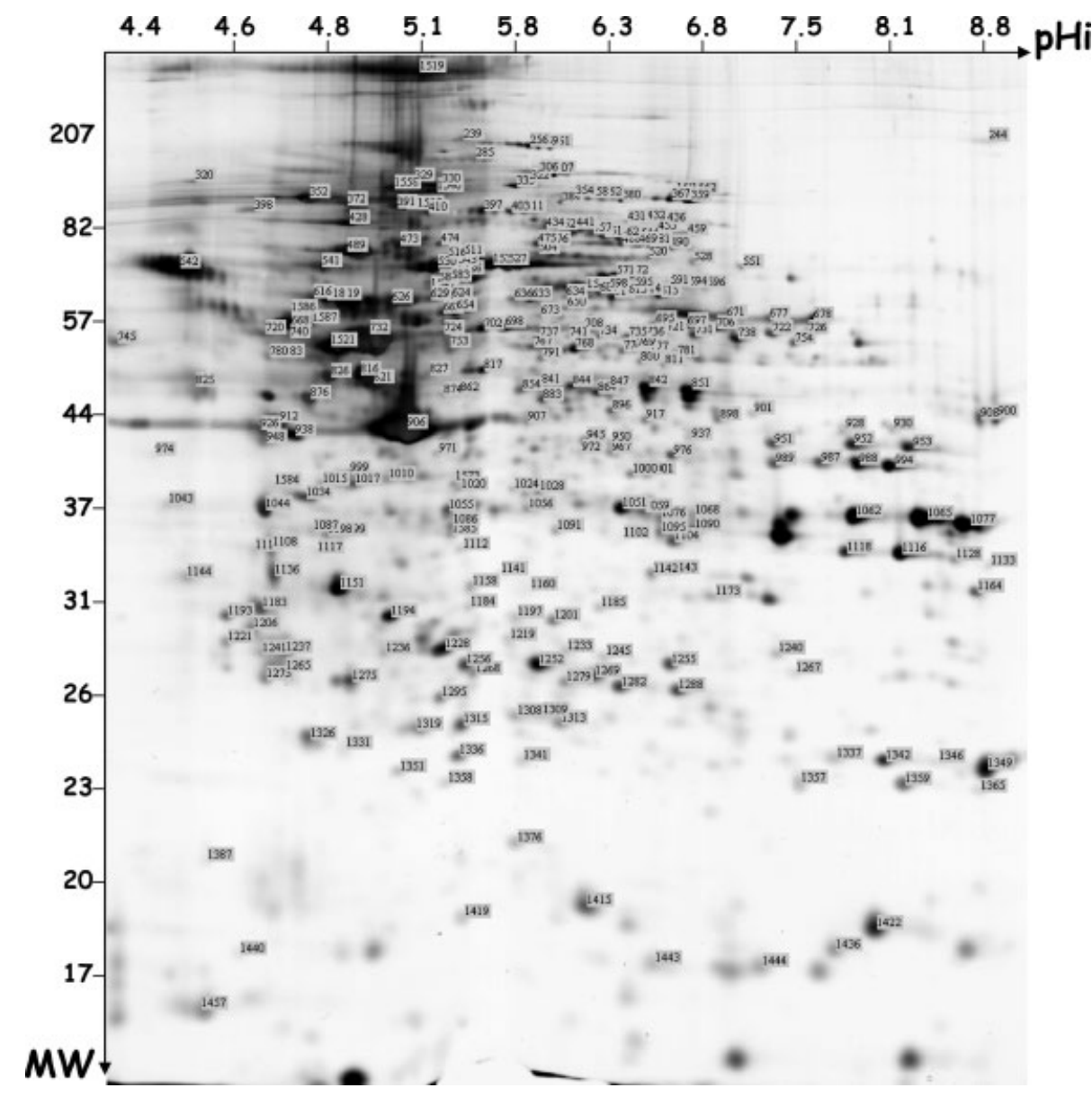

Figure 1. 2-DE master gel of BMMSC. Human adult primary BMMSC were separated using 2-DE electrophoresis in a dry strip $\mathrm{pH}$ 3-10 for the first dimension, a $12 \%$ SDS-PAGE for the second dimension and silver stained. The identification of proteins, noted with their serial number, was performed by PMFs after trypsin digestion and MALDITOF on Coomassie-stained spots. 

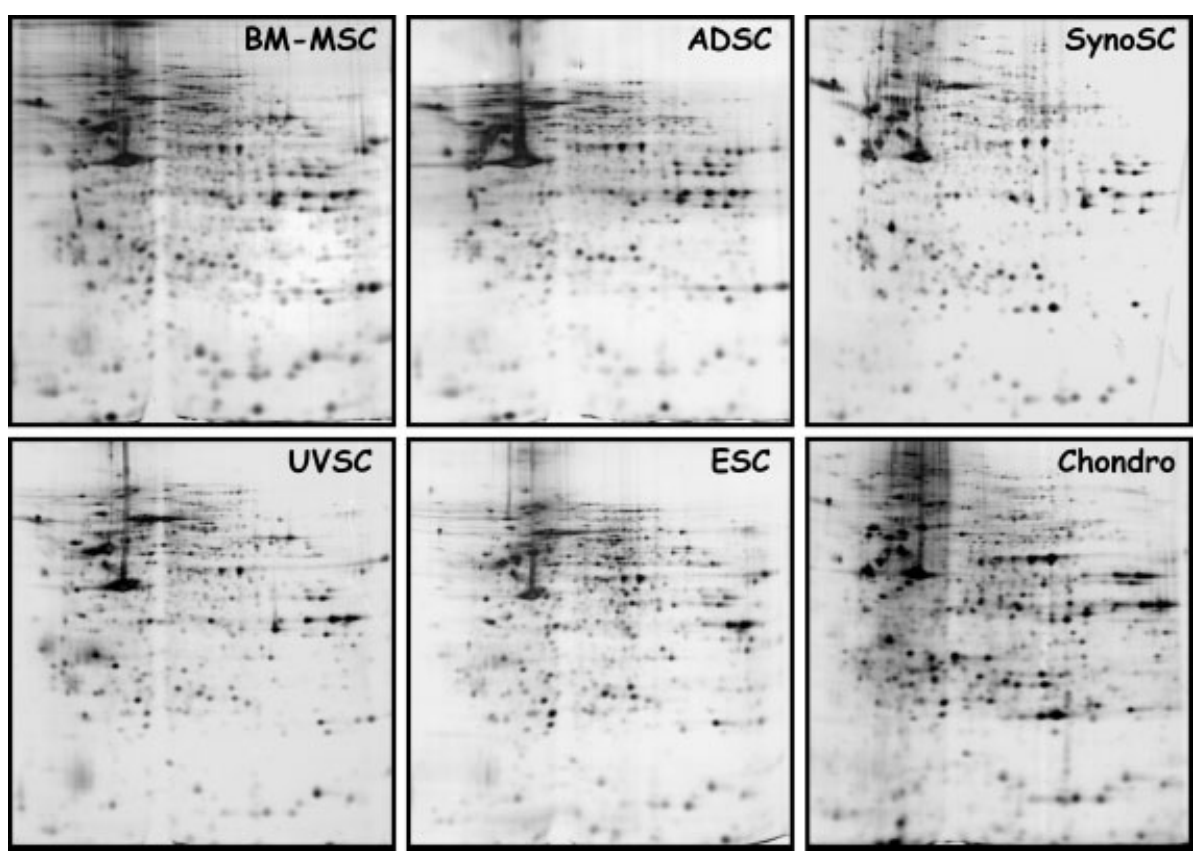

Figure 2. Representative 2-DE gels of the different cultures. Gels were obtained from BMMSC, ADSC, UVSC, SynoSC, hESC and primary human chondrocyte cultures. Whole cell extracts $(30 \mu \mathrm{g})$ were separated using 2-DE electrophoresis in a dry strip pH 3-10 for the first dimension and a $12 \%$ SDS-PAGE for the second and silver stained. The gel is representative of 3-5 gels from independent cultures samples performed before statistical analysis.

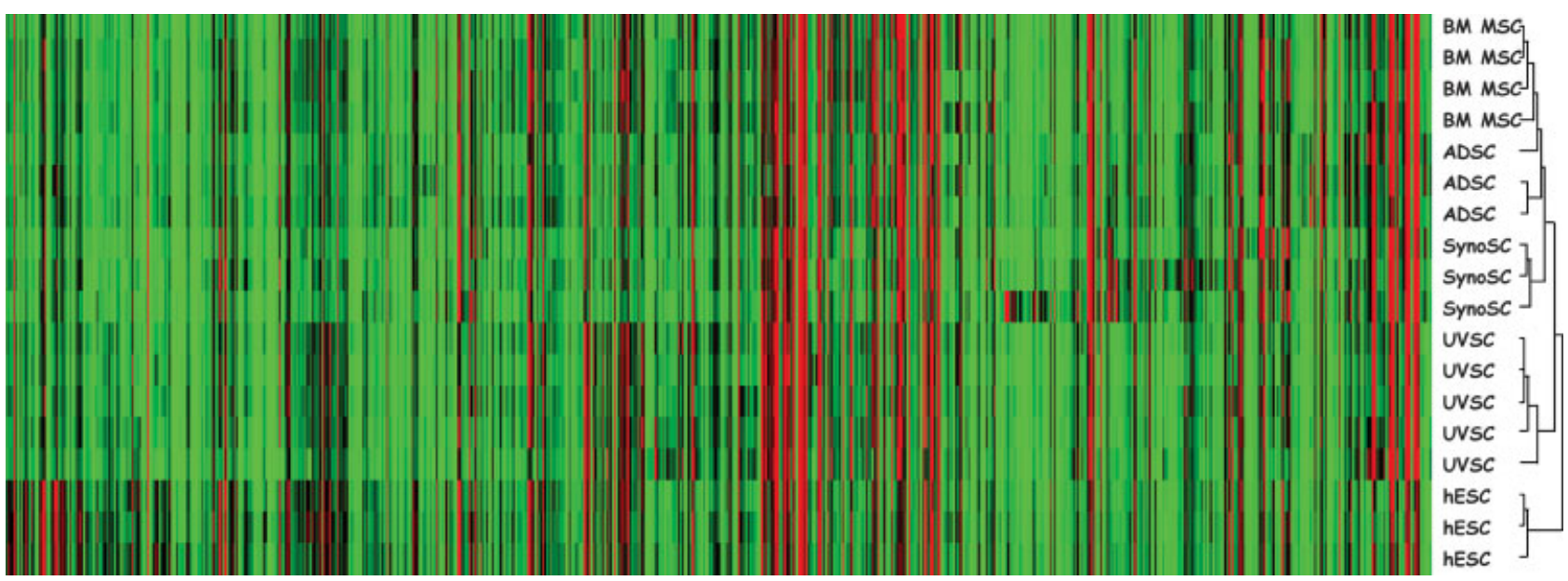

Figure 3. Hierarchical clustering of the 2-DE data. Data from BM-MSC, ADSC, UVSC, SynoSC and hESC have been generated by comparison, quantification and normalization of the different 2-DE gel using Progenesis SameSpot. Clustering was performed using the Hierarchical Clustering Explorer version 3 software with Manhattan Distance as statistical test.

of hESC. Interestingly, BM-MSC and ADSC were very closely related in this analysis while 'adult' stem cells including BMMSC, ADSC and SynoSC could be segregated from 'foetal' UVSC.

The variability of the spots between replicates for most stem cells was close to $30 \%$, which was satisfactory. Spots with significant modified expression were selected based on an increase or decrease expression by a factor of 1.7 or a $p$ value inferior at 0.05 . The $p$-value was calculated by two methods, $t$-test in order to compare each type of cells to BMMSC and ANOVA to compare all stem cells together. The percentage of spots with a significant modification of expression, when compared to BM-MSC, were 22, 40, 22 and
48 for UVSC, SynoSC, ADSC and hESC, respectively. When limited to identified proteins, the numbers were 16, 25, 14 and $46 \%$ showing that the sampling for identification was representative of all the spots detected in the gels.

To illustrate differences in proteomic patterns, the number of common and significantly different spots between BM-MSC, ADSC and SynoSC, as well as, between BM-MSC, UVSC and hESC were computed (Fig. 4). From this general analysis, BM-MSC, ADSC and SynoSC appeared very close with an high percentage of common spots $(68.5 \%)$ and a low number of cell specific ones (BM-MSC: $5.5 \%$, ADSC: $4 \%$, SynoSC: $14.2 \%)$. BM-MSC and ADSC had also more spots in common $(98+336)$ than all the other MSC related cells. 
When BM-MSC were compared to UVSC and hESC, a high number of spots was present in only one cell type (60 for BMMSC, 38 for UVSC and 169 for hESC). As expected, hESC were more apart than BM-MSC, ADSC and SynoSC. Importantly, these differences for detected spots were also analysed at the level of identified proteins. It was then possible to group proteins that were specific for MSC-like cells (common to BM-MSC, ADSC and SynoSC) versus hESC (Table 2).

This selection was the basis for a proposed proteomic signature of human MSC (see Section 4). In order to evaluate the specificity of this signature, we compared the 2-D expression of the selected proteins in MSC and in human primary chondrocytes. Proteins which are different between these two types of cells are indicated by a star in the Table 2 and shown in Table 4 of Supporting Information.

\subsection{Functional block analysis}

To analyse in more details proteomic differences, cell functions were associated to identified proteins using the SwissProt (http://www.expasy.org/) and the Gene Ontology (http://www.geneontology.org/) databases (Table 2 of Supporting Information). The percentage of proteins with a differential expression between BM-MSC and ADSC, SynoSC, UVSC or hESC is plotted in different functional blocks (Fig. 5). hESC were clearly distinguishable from the other cells in particular for function related to 'energy and ROS' and 'metabolism'. In the group 'protein synthesis and proteolysis' that was represented by 61 different proteins, both SynoSC and hESC had a distinct pattern which was in agreement with the clustering analysis (Fig. 3). The Ingenuity software (www.ingenuity.com) which is able to perform a Fisher statistical comparison between functional blocks was also employed. A dataset containing proteins identifiers and corresponding expression values was uploaded into the application. Each protein identifier was mapped to its corresponding object in the Ingenuity Pathways Knowledge Base. A fold increase cut-off of 1.7 and $p$-value cut-off of 0.05 were set to identify genes whose expression was significantly differentially regulated. These proteins, called focus proteins, were overlaid onto a global molecular network developed from information contained in the Ingenuity Pathways
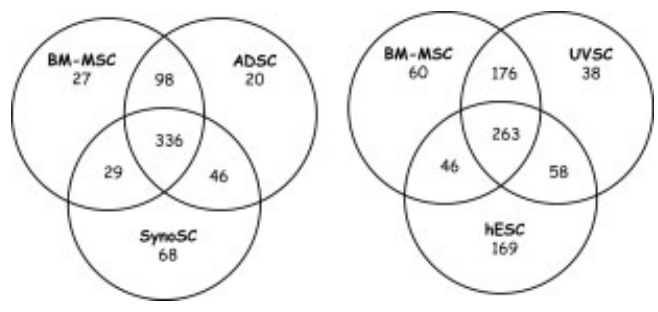

Figure 4. Common and significantly different 2-DE proteins. The numbers correspond to the spots of proteins from the master gel in common or significantly different between BM-MSC, ADSC and ECS (left panel) and BM-MSC, UVSC and SynoSC (right panel).
Knowledge Base. Networks of these focus proteins were then algorithmically generated based on their connectivity. The analysis identified clear functional differences between the cell types (Fig. 6). SynoSC and hESC appeared the distant from other cells. This was particularly apparent for the 'carbohydrate metabolism', 'amino acid metabolism' and 'cell death' groups for the hESC and for the 'PTM' and the 'protein folding' groups for the SynoSC. In the latter cells the expression of TCP-1 $\alpha, \beta, \varepsilon$, HSP70 and HSP90 was remarkable.

\section{Discussion}

In this study, cultured undifferentiated human MSC isolated from BM-MSC, UVSC, ADSC and SynoSC were analysed by 2-DE. The first task was to generate a comprehensive proteomic description of human primary BM-MSC. These cells were then compared with the other 'MSC like' cells and with pluripotent hESC. The main goal of this study was to link the proteomic patterns of the cells to tissue origin and phenotypic properties. This was a way to figure out if all sources of MSC were equivalent, and if a specific human MSC proteomic signature could be generated (Table 2).

While the first proteomic analysis of MSC was performed and published in 2001 by Prockop and coworkers [10], not many studies explored in detail the whole proteome of MSC cells (for review see ref. [18]). Based on the published articles, it was not possible to identify a clear proteomic signature specific for MSC. One of the reasons was the different paradigms, protein extraction protocols and analytical approaches used in the different studies. Through the European Genostem Consortium (www.ge nostem.org), it was possible to generated proteomic samples from MSC isolated from various tissues using an optimized standard protocol (medium, extraction buffer and procedure). The cells used as reference in this study were human BM-MSC since they were the most commonly used MSC. To facilitate proteomic comparisons, a BM-MSC master gel was constructed with an important number of proteins identified by MS. The later were distributed in a broad range of pHi (Fig. 1 of Supporting Information), belonged to most cellular compartments (cytoplasm, nucleus, mitochondrion, reticulum, golgi and membrane) and represented a wide range of functions (from glycolysis to splicing and signal transduction) (Table 2 of Supporting Information). The list of identified proteins was consistent with previous proteomic investigations with the following common proteins identified: annexin V, TCP1 $\alpha$, lactate dehydrogenase $\mathrm{B}$, phosphoglycerate kinase 1 [10], programmed cell death 6 interacting protein, peroxiredoxin 2, glucose-6-phosphate 1-dehydrogenase, pyruvate kinase M1/ M2 isozyme, annexin A2 [21] or hnRNP A2/B1 [12, 22, 23]. Importantly, these proteins were originally identified following differential studies in subpopulations of MSC, whereas in our case they were expressed in BM-MSC. 
Table 2. List of identified proteins with differential expression BM-MSC and ADSC by comparison with hESC

\begin{tabular}{|c|c|c|c|c|}
\hline ID & Protein & Accession no. & Fold ratio & $t$-Test $(p)$ \\
\hline 505 & Adenine phosphoribosyltransferase & P07741 & 2.5 & 0.001 \\
\hline 623 & Adenylyl cyclase-associated 1 & Q01518 & -1.5 & 0.008 \\
\hline 529 & Adenylyl cyclase-associated 1 & Q01518 & 2.2 & 0.002 \\
\hline 758 & ${ }^{*}$ Annexin A1 & P04083 & -1.8 & 0.005 \\
\hline 567 & ${ }^{*}$ Annexin A1 & P04083 & -1.3 & 0.231 \\
\hline 912 & ${ }^{*}$ Annexin A2 & P07355 & -3.5 & 0.001 \\
\hline 606 & Annexin A2 & P07355 & -5.4 & $<0.001$ \\
\hline 125 & ATP synthase D & 075947 & 2.3 & $<0.001$ \\
\hline 445 & ${ }^{*}$ Caldesmon & Q9NYG1 & -2.6 & 0.010 \\
\hline 926 & ${ }^{*}$ Caldesmon & Q9NYG1 & -3.3 & 0.001 \\
\hline 625 & ${ }^{*}$ Caldesmon & Q9NYG1 & -2.2 & 0.028 \\
\hline 956 & ${ }^{*}$ Caldesmon & Q9NYG1 & -3.1 & 0.001 \\
\hline 569 & Calreticuline & P27797 & -2.4 & 0.007 \\
\hline 977 & Calumenin & 043852 & -2.7 & 0.003 \\
\hline 980 & ${ }^{*}$ Chloride intracellular channel protein 1 & 000299 & -1.4 & 0.006 \\
\hline 160 & Chloride intracellular channel protein 4 & Q9Y696 & -1.9 & 0.007 \\
\hline 976 & Cytosol aminopeptidase & P28838 & 1.9 & 0.007 \\
\hline 561 & $E F-1-\delta$ & P29692 & -2.2 & 0.001 \\
\hline 536 & $\mathrm{EF}-1-\delta$ & P29692 & -1.8 & 0.002 \\
\hline 292 & $\mathrm{EF}-1-\delta$ & P29692 & -1.0 & 0.981 \\
\hline 297 & Elongation factor $\tau$ & P49411 & 2.6 & $<0.001$ \\
\hline 626 & F-actin capping protein $\beta$ & P47756 & 2.0 & 0.001 \\
\hline 953 & F-actin capping protein $\beta$ spot 1 & P47756 & 1.4 & 0.011 \\
\hline 80 & Fumarate hydratase & P07954 & 2.3 & 0.005 \\
\hline 332 & FUSE-binding protein 1 & Q96AE4 & 2.7 & 0.002 \\
\hline 402 & FUSE-binding protein 1 & Q96AE4 & 4.0 & $<0.001$ \\
\hline 366 & ${ }^{*}$ FUSE-binding protein 2 & Q5U4P6 & 2.9 & $<0.001$ \\
\hline 409 & FUSE-binding protein 2 & Q5U4P6 & 2.3 & 0.049 \\
\hline 512 & FUSE-binding protein 2 & Q5U4P6 & 2.3 & 0.085 \\
\hline 601 & Glucose-6-phosphate 1-dehydrogenase & P11413 & 1.6 & 0.049 \\
\hline 589 & Glucose-6-phosphate 1-dehydrogenase & P11413 & 1.9 & $<0.001$ \\
\hline 532 & GST P & P09211 & 2.7 & 0.001 \\
\hline 494 & GST P & P09211 & 1.4 & 0.005 \\
\hline 461 & Glutathione transferase $\omega-1$ & P78417 & 1.2 & 0.135 \\
\hline 603 & Glutathione transferase $\omega-1$ & P78417 & -1.1 & 0.646 \\
\hline 866 & hn RNPL & P14866 & 1.5 & 0.369 \\
\hline 503 & hn RNP L & P14866 & 2.4 & 0.002 \\
\hline 538 & hn RNP L & P14866 & 4.7 & 0.002 \\
\hline 885 & hnRNP H & P31943 & 2.3 & 0.002 \\
\hline 562 & HSP27 $\beta$ & P04792 & -1.8 & 0.013 \\
\hline 580 & ${ }^{*} \mathrm{HSP} 27 \beta$ & P04792 & -2.6 & 0.007 \\
\hline 519 & *Inorganic pyrophosphatase & Q15181 & 3.3 & $<0.001$ \\
\hline 757 & Iron-responsive element-binding 1 & P21399 & 1.9 & 0.114 \\
\hline 848 & *Isocitrate dehydrogenase [NADP] & 075874 & 4.2 & 0.005 \\
\hline 616 & Lactoylglutathione lyase & 004760 & 3.5 & $<0.001$ \\
\hline 975 & L-lactate dehydrogenase B & P07195 & 2.0 & 0.002 \\
\hline 386 & Microtubule-associated protein 1B & $\mathrm{P} 46821$ & -1.8 & 0.047 \\
\hline 257 & Nucleophosmin & P06748 & 2.0 & $<0.001$ \\
\hline 376 & Peroxiredoxin 4 & Q13162 & -1.1 & 0.634 \\
\hline 476 & Peroxiredoxin 6 & P30041 & 1.9 & 0.002 \\
\hline 583 & Peroxiredoxin 1 & 006830 & 2.4 & $<0.001$ \\
\hline 802 & Peroxiredoxin 2 & P32119 & 2.5 & $<0.001$ \\
\hline 57 & *Poly $(\mathrm{RC})$ binding protein 1 & Q15365 & 2.6 & $<0.001$ \\
\hline 573 & Poly $(\mathrm{RC})$ binding protein 1 & Q15365 & 1.5 & 0.033 \\
\hline 314 & Poly $(\mathrm{RC})$ binding protein 2 & Q68Y55 & 2.0 & 0.058 \\
\hline 984 & Proliferating cell nuclear antigen & P12004 & 2.1 & 0.001 \\
\hline
\end{tabular}


Table 2. Continued

\begin{tabular}{lllrr}
\hline ID & Protein & Accession no. & Fold ratio & $t$-Test $(p)$ \\
\hline 493 & Proteasome subunit $\alpha$ type 1 & P25786 & 1.9 & 0.010 \\
398 & Proteasome subunit $\alpha$ type 3 & P25788 & 1.4 & 0.027 \\
944 & Protein disulphide isomerase A1 & P07237 & -2.5 & $<0.001$ \\
619 & Pyruvate dehydrogenase E1 & P11177 & 1.9 & 0.004 \\
819 & Ras-related protein Rab-11A & P62491 & 2.6 & 0.003 \\
400 & Stomatin-like protein 2 & Q9UJZ1 & 2.1 & 0.002 \\
\hline
\end{tabular}

* Indicate proteins with differential expression in human primary chondrocyte.

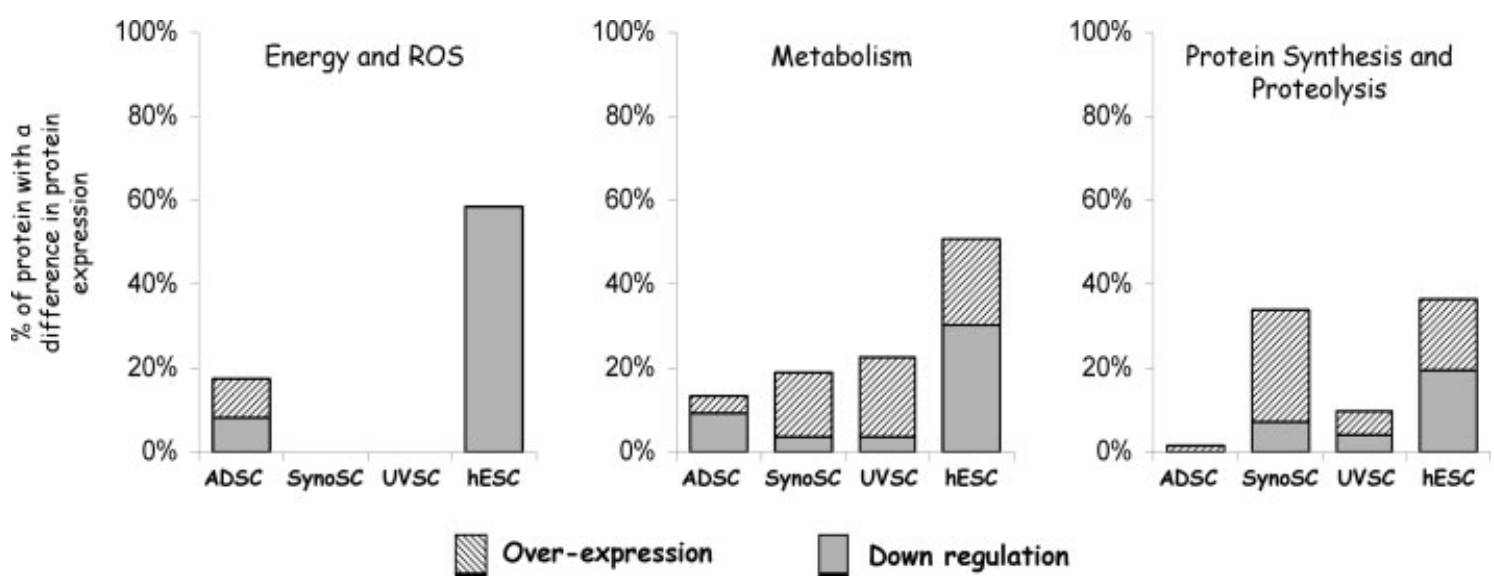

Figure 5. Functional block analysis. Proteins with differential expression (up- or downregulated) were distributed between functional blocks using the Swiss-Prot (http://www.expasy.org/) and the Gene Ontology (http:// www.geneontology.org/) databases. Three main blocks were represented: proteins involved in 'energy and ROS' (left panel, 12 protein spots), in 'metabolism' (middle panel, regrouping 54 proteins involved in 'glycolysis and carbohydrate metabolism', 'metabolism and catabolism' and 'synthesis of metabolites'), and in 'protein synthesis and proteolysis' (right panel, 54 proteins spot, contains also the 'folding and heat shock protein' block).

One important goal of this study was to use proteomics to compare MSC isolated from different tissues. Since the different types of culture were provided by different laboratories, biases were reduced by using in all places the same batches of medium, the same procedures and buffers for the preparation of the proteomic extracts. The heterogeneity related to the fact that the cultures were produced in different laboratories was therefore minimized and almost irrelevant since the cell types (ADSC, UVSC, etc.) were anyway generated from different origins and cultured in different conditions (Table 1 of Supporting Information). The different culture methods ensured the preservation of MSC characteristics in terms of differentiation properties (osteoblastic, adipocytic and chondrogenic pathways; data not shown) and expression of major phenotypic markers (Table 1). As an additional control of the impact of the production of the cells in different laboratories, BM-MCS produced in different participating laboratories were indistinguishable (data not shown and [24]).

Although BM-MSC, ADSC, SynoSC and UVSC share common capacities of differentiation into osteoblast, chondrocyte and adipocyte, several studies have already demonstrated differences between these cells in terms of pro- liferating rate, senescence or differentiation capability in specific pathways [4, 13]. Importantly, while CD expression patterns (Table 1) were not sufficient to differentiate them, our global proteomic approach could clearly achieve this goal (Fig. 3). Our data also suggested that among MSC related cells, ADSC were the closest match to BM-MSC, while the UVSC were the more distant. This conclusion was not only based on the clustering experiments, but also on the functional block analysis and on the number of proteins with differential expression among cells (Figs. 4-6). Our result gives additional basis for the use of ADSC as an alternative to BM-MSC for regenerative medicine, these two types of cells being very close in comparison with the others. It appeared also in the functional analysis (Fig. 6), that SynoSC were quite distant to BM-MSC which was also a conclusion of the genomic analysis of these cells [4].

We also compared MSC cells to hESC. The latter were cultured in a specific medium without the use of feeder cells to facilitate the proteomic analysis (Table 1 of Supporting Information). As mentioned above, differences in culture media could be responsible for proteomic differences, but cannot be avoided to compare cells with various physiologi- 


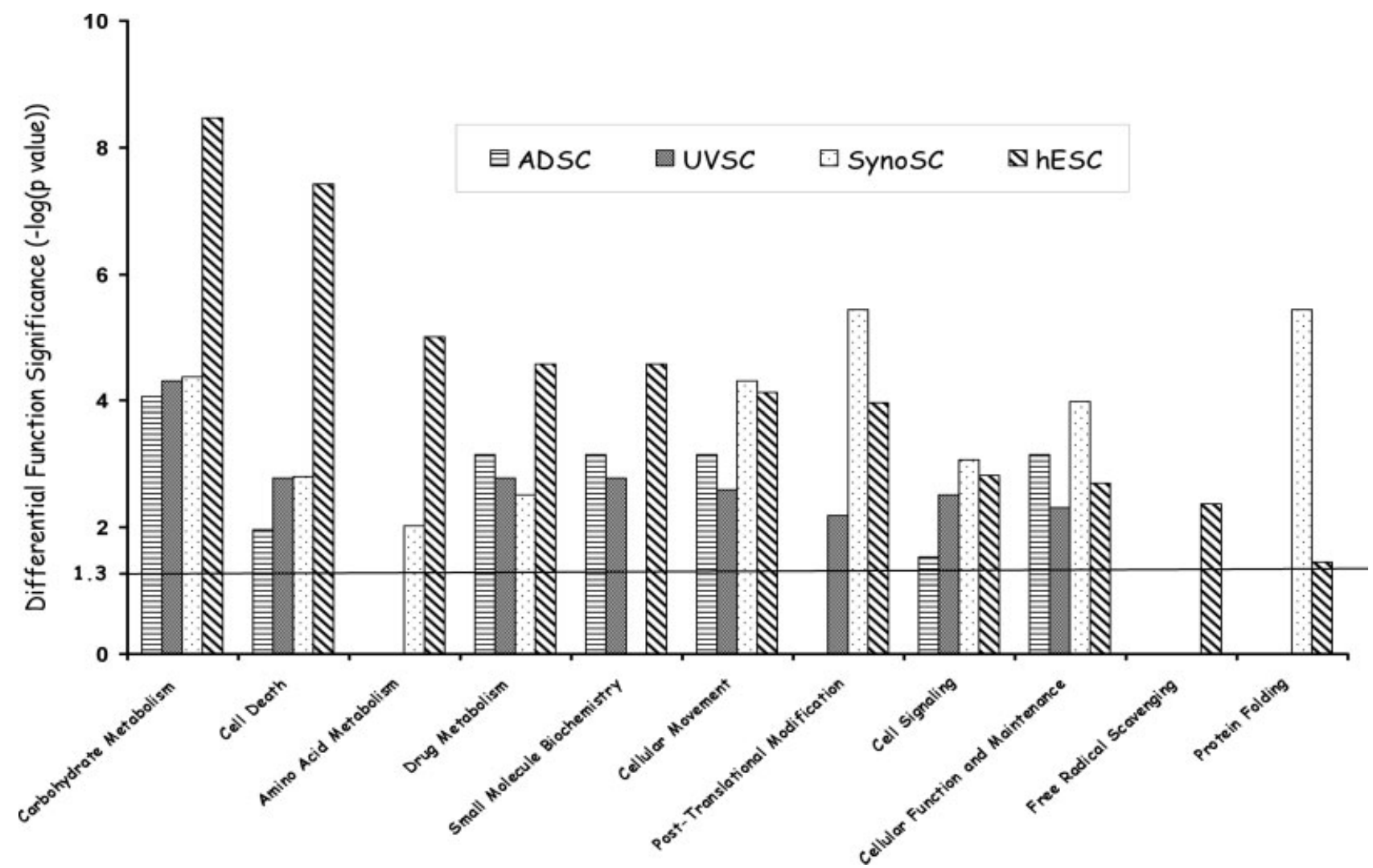

Figure 6. Statistical analysis of differential function. The Ingenuity Software was used to identify differential functions between the various stem cells. In the $y$-axis are plotted the statistical values (as minus log of $p$ value) obtained from the comparison with the BM-MSC reference. In the $x$-axis are indicated the different functional networks defined by the Ingenuity analysis.

cal characteristics and phenotypes. Global or functional analysis (Figs. 3-6) revealed that hESC were clearly distinct from BM-MSC, and from the other cell lines studied. BMMSC, ADSC, SynoSC and UVSC could therefore be regrouped in an 'MSC-like group' with the UVSC being the closest to hESC; this might be related to their foetal origin. The Ingenuity software analysis, which represents an original approach to construct networks of function and calculate a $p$-value related to their modification, confirmed this analysis pointing out differences in particular in the 'carbohydrate metabolism', 'amino acid metabolism' and 'cell death' functional blocks (Fig. 6). The latter group was interesting since it is in relation with stem cell maintenance, self renewal and differentiation during the development [25]. Some individual proteins in this pathways (Table 2) have already been pointed out in others differential proteomic studies. CLIC4 a mitochondrion chloride channel involved in myc induced apoptosis [26], was already identified as having a lower expression in hESC and in TERT-modified MSC [23]. The same observation might be done for annexin A1, A2 which were downregulated in TERT-modified MSC [23] and in our results compared with hESC. In addition, elongation factor TU, peroxiredoxin 1 and 6 were upregulated in both TERT-modified MSC [23] and in hESC. This ingenuity-based analysis renders possible the participation of proteins to different functional blocks which makes sense knowing the multiple relations and functions of proteins.

To progress towards the definition of a human MSC signature, proteins whose expression was similar in BM-MSC, ADSC and SynoSC, but significantly different in hESC, were selected (Table 2, and Tables 2-4 of Supporting Information). Proteins from most functional blocks were present in this selection which suggested that differences between stem cells were more global than focussed. In this list, we selected several proteins which could define a small panel of proteins specific for MSC and hESC. Importantly, we could validate this list by selecting the proteins (Table 2, indicated by a star) which were with differential expression in human primary chondrocyte. Overall, we could select as markers of MSC: annexin A1 and A2 (ANXA1 and ANXA2) and HSP27 $\beta$ (HSPB1). For hESC, elongation factor Tu (TUFM), isocitrate dehydrogenase (IDH1) and the peroxiredoxin 1, 2, and 6 (PRDX1, PRDX2, PRDX6) were the most interesting ones. These proteins are likely to be involved in the different phenotypes and capacities for differentiation.

In conclusion, the proteomic investigation of human mesenchymal and embryonic stem cells realized in this study is complementary to previous proteomics and geno- 
mics works [18]. The challenge in these broad types of approaches is to identify, in the huge amount of data generated, relevant differences and similarities that will help characterizing stem cells and linked phenotypes with molecular markers. The generation of a master 2-D gel from human BM-MSC, combined with the analysis of the other stem cells adds a lot to the general proteomic knowledge of MSC. In this work, we demonstrated that a proteomic 'MSClike' profile can be defined. Interestingly, umbilical vein wall stem cells, a neo-natal cell type, belonged to this group, and were closer to MSC than to hESC. In addition, we confirmed that BM and adipose tissue derived cells are very similar which argue for their interchangeable use in therapy. Finally, we proposed a panel of proteins which is the basis for the definition of a human MSC proteomic signature that would need further validation on additional cell types.

This work was supported by the European Commission through the FP6 project GENOSTEM (LHSB-CT-2003503161).

The authors have declared no conflict of interest.

\section{References}

[1] Pittenger, M. F., Mackay, A. M., Beck, S. C., Jaiswal, R. K. et al., Multilineage potential of adult human mesenchymal stem cells. Science 1999, 284, 143-147.

[2] Zuk, P. A., Zhu, M., Mizuno, H., Huang, J. et al., Multilineage cells from human adipose tissue: Implications for cell-based therapies. Tissue Eng. 2001, 7, 211-228.

[3] De Bari, C., Dell'Accio, F., Tylzanowski, P., Luyten, F. P., Multipotent mesenchymal stem cells from adult human synovial membrane. Arthritis Rheum. 2001, 44, 1928-1942.

[4] Djouad, F., Bony, C., Haupl, T., Uze, G. et al., Transcriptional profiles discriminate bone marrow-derived and synoviumderived mesenchymal stem cells. Arthritis Res. Ther. 2005, 7, R1304-R1315.

[5] Lee, O. K., Kuo, T. K., Chen, W. M., Lee, K. D. et al., Isolation of multipotent mesenchymal stem cells from umbilical cord blood. Blood 2004, 103, 1669-1675.

[6] Alsalameh, S., Amin, R., Gemba, T., Lotz, M., Identification of mesenchymal progenitor cells in normal and osteoarthritic human articular cartilage. Arthritis Rheum. 2004, 50, 15221532.

[7] Kaltz, N., Funari, A., Hippauf, S., Delorme, B. et al., In vivo osteoprogenitor potency of human stromal cells from different tissues does not correlate with expression of POU5F1 or its pseudogenes. Stem Cells 2008, 26, 2419-2424.

[8] Fukuda, K., Development of regenerative cardiomyocytes from mesenchymal stem cells for cardiovascular tissue engineering. Artif. Organs 2001, 25, 187-193.

[9] Yamanaka, S., Li, J., Kania, G., Elliott, S. et al., Pluripotency of embryonic stem cells. Cell Tissue Res. 2008, 331, 5-22.

[10] Colter, D. C., Sekiya, I., Prockop, D. J., Identification of a subpopulation of rapidly self-renewing and multipotential adult stem cells in colonies of human marrow stromal cells. Proc. Natl. Acad. Sci. USA 2001, 98, 7841-7845.
[11] Feldmann, R. E., Jr., Bieback, K., Maurer, M. H., Kalenka, A. et al., Stem cell proteomes: A profile of human mesenchymal stem cells derived from umbilical cord blood. Electrophoresis 2005, 26, 2749-2758.

[12] Delany, J., Floyd, Z. E., Zvonic, S., Smith, A. et al., Proteomic analysis of primary cultures of human adipose derived stem cells: Modulation by adipogenesis. Mol. Cell. Proteomics 2005, 4, 731-740.

[13] Izadpanah, R., Trygg, C., Patel, B., Kriedt, C. et al., Biologic properties of mesenchymal stem cells derived from bone marrow and adipose tissue. J. Cell. Biochem. 2006, 99, 12851297.

[14] Im, G. I., Shin, Y. W., Lee, K. B., Do adipose tissue-derived mesenchymal stem cells have the same osteogenic and chondrogenic potential as bone marrow-derived cells? Osteoarthritis Cartilage 2005, 13, 845-853.

[15] Puissant, B., Barreau, C., Bourin, P., Clavel, C. et al., Immunomodulatory effect of human adipose tissue-derived adult stem cells: Comparison with bone marrow mesenchymal stem cells. Br. J. Haematol. 2005, 129, 118-129.

[16] Sakaguchi, Y., Sekiya, I., Yagishita, K., Muneta, T., Comparison of human stem cells derived from various mesenchymal tissues: Superiority of synovium as a cell source. Arthritis Rheum. 2005, 52, 2521-2529.

[17] Wagner, W., Wein, F., Seckinger, A., Frankhauser, M. et al., Comparative characteristics of mesenchymal stem cells from human bone marrow, adipose tissue, and umbilical cord blood. Exp. Hematol. 2005, 33, 1402-1416.

[18] Roche, S., Provansal, M., Tiers, L., Jorgensen, C., Lehmann, S., Proteomics of primary mesenchymal stem cells. Regenerat. Med. 2006, 1, 511-517.

[19] Shevchenko, A., Wilm, M., Vorm, O., Mann, M., Mass spectrometric sequencing of proteins silver-stained polyacrylamide gels. Anal. Chem. 1996, 68, 850-858.

[20] Seo, J., Bakay, M., Chen, Y. W., Hilmer, S. et al., Interactively optimizing signal-to-noise ratios in expression profiling: Project-specific algorithm selection and detection p-value weighting in Affymetrix microarrays. Bioinformatics 2004, 20, 2534-2544.

[21] Wang, D., Park, J. S., Chu, J. S., Krakowski, A. et al., Proteomic profiling of bone marrow mesenchymal stem cells upon TGF-beta stimulation. J. Biol. Chem. 2004, 279, 4372543734.

[22] Park, H. W., Shin, J. S., Kim, C. W., Proteome of mesenchymal stem cells. Proteomics 2007, 7, 2881-2894.

[23] Huang, G. P., Pan, Z. J., Huang, J. P., Yang, J. F. et al., Proteomic analysis of human bone marrow mesenchymal stem cells transduced with human telomerase reverse transcriptase gene during proliferation. Cell Prolif. 2008, 41, 625-644.

[24] Kastrinaki, M. C., Sidiropoulos, P., Roche, S., Ringe, J. et al., Functional, molecular and proteomic characterization of bone marrow mesenchymal stem cells in rheumatoid arthritis. Ann. Rheum. Dis. 2007, 67, 741-749.

[25] Trioulier, Y., Torch, S., Blot, B., Cristina, N. et al., Alix, a protein regulating endosomal trafficking, is involved in neuronal death. J. Biol. Chem. 2004, 279, 2046-2052.

[26] Shiio, Y., Suh, K. S., Lee, H., Yuspa, S. H. et al., Quantitative proteomic analysis of myc-induced apoptosis: A direct role for Myc induction of the mitochondrial chloride ion channel, mtCLIC/CLIC4. J. Biol. Chem. 2006, 281, 2750-2756. 